\title{
Vertical-cavity semiconductor devices for fluorescence spectroscopy in biochips and microfluidic platforms
}

\author{
P. A. Porta \\ H. D. Summers \\ Cardiff University \\ School of Physics and Astronomy \\ 5, The Parade \\ Cardiff, CF24 3YB Wales, United Kingdom
}

\begin{abstract}
This paper describes the properties of vertical-cavity semiconductor devices designed to emit light when driven in forward bias mode and detect optical radiation at wavelengths longer than that of emission when driven in reverse bias mode. The study of this type of devices is motivated by the miniaturization and integration into a single unit of the three functions that a microfluorimeter has to perform, optical pumping, optical detection, and optical filtering of weak light sources. The devices produced can generate fluorescence with a low output power since their emission wavelength can be tuned with that of maximum absorption of the fluorescent dye. We demonstrate also that they can detect low power fluorescence generated in a small volume of concentrated solution of a commercial dye. These devices can find useful application in microanalytical systems such as microfluidic devices or optical biochips. () 2005 Society of Photo-Optical Instrumentation Engineers. [DOl: 10.1117/1.1925229]
\end{abstract}

Keywords: vertical-cavity semiconductors; spectroscopy; microfluorimeters; optical pumping; optical detection; optical filtering.

Paper 04213R received Nov. 8, 2004; revised manuscript received Jan. 20, 2005; accepted for publication Feb. 3, 2005; published online May 23, 2005.

\section{Introduction}

Biochips are commonly defined as microdevices designed to perform biochemical procedures for biomedical applications rapidly and inexpensively in a miniaturized environment. ${ }^{1}$ These systems with their integrated detection capabilities have great potential for use in experimental genomics, in proteomics, and by doctors at the point of care. ${ }^{1}$ Microfluidics platforms are miniaturized chemical analysis systems that allow for the transport of the analyte in microchannels, its chemical separation into its various components, and their detection with electrochemical or optical sensors. ${ }^{2}$

Fluorescence spectroscopy has become the most commonly used technique in these two types of microanalysis systems for its high sensitivity, for its selectivity of the products $^{1,3}$ and because it is chemically decoupled from the chemical and biological processes in the microchip. ${ }^{4}$ Arc and incandescent lamps are commonly used to generate fluorescence emission because of their broadband continuous emission $^{5}$ but their size, their low efficiency, and their low stability ${ }^{6}$ do not make them suitable for miniaturized portable analysis systems. Light-emitting diodes (LEDs) on the contrary are compact, robust, highly efficient, highly stable optical radiation sources and are available in a wide range of

Address all correspondence to Piepaolo Porta, Physics and Astronomy, Cardiff University, 5, The Parade, Cardiff, Wales CF24 3YB United Kingdom, Tel: 004429-20875044; Fax: 0044-29-20874056; E-mail: portapa@cf.ac.uk wavelengths, from ultraviolet down to the infrared part of the optical spectrum. ${ }^{7}$

In the 1980s, when the first blue LEDs became available, it was demonstrated that it was possible to use these devices as excitation sources for fluorescence detection in an acid-base titrator. ${ }^{8}$ A few years later a high-precision compact fluorimeter was built, capable of detecting fluorophores concentrations as low as few nanograms per liter. ${ }^{6}$ Today LEDs are widely used in chemistry and biochemistry for absorbance, fluorescence, and spectroelectrochemical measurements as illustrated by a review paper about their use in a miniaturized analytical device such as a planar flowthrough cell. ${ }^{9}$ A LED has also been utilized as a nanosecond ultraviolet light source to measure the fluorescence decay of a quinine-sulfate solution, whose lifetime $\tau$ was $19.5 \mathrm{~ns} .{ }^{10}$ The technique of measuring directly the fluorescence lifetime following the excitation pulse can only be used with fluorescent samples whose decay time is much longer than that of the pulsed LED. The phase modulation technique instead allows for measurement in the frequency domain of nanosecond fluorescence lifetimes, ${ }^{5}$ comparable if not shorter than that of the excitation source. A single quantum well light emitting diode was utilized with excellent results in this type of instrumentation ${ }^{11,12}$ and the authors of Ref. 11 were able to measure fluorescence lifetimes of about $3 \mathrm{~ns}$.

$1083-3668 / 2005 / \$ 22.00$ @ 2005 SPIE 
A further step towards the realization of portable fluorimeters was the miniaturization of the detection system and of the optical filtering stage used to reject emission and scattering from the excitation source. An example of these cases can be found in Ref. 13 where a $30 \mu \mathrm{m}$ microavalanche photodiode was placed underneath a $50 \mu \mathrm{m}$ microfluidic channel fabricated in poly(dimethylsiloxane). A $80-\mu \mathrm{m}$-thick yellow polycarbonate rejection filter was mounted between the microchannel and the photodiode and illumination of the sample was obtained with an optical fiber from the side of the microchannel. The authors of Ref. 4 instead fabricated a silicon photodiode in a silicon substrate, grew a thin film interference filter on the top of the wafer, and built an $200 \mu \mathrm{m}$ wide electrophoresis microchannel system above them with parylene, a particular plastic to provide chemical insulation between the flowing ions and the detector below.

There are different techniques for the integration of optoelectronic devices on a common substrate, such as hybrid technology, flip-chip mounting or epitaxial lift-off. ${ }^{14}$ A LED can be transferred with lift-off processes from its natural substrate to a silicon substrate ${ }^{15}$ where the photodetector can be patterned with standard techniques. The LED sits on the same side of the photodetector and emits light away from its surface to excite fluorescent samples placed in front of them. The fluorescence emission that is collected by the detector propagates in the direction opposite to that of excitation source output and the amount of the scattered light that reaches the photodiode can be significantly reduced. ${ }^{15}$ Several and complex processing steps are required to transfer the LED from its native surface to the silicon substrate and problems like mismatch of the thermal expansion coefficients of the two heterogeneous materials can affect the lifetime and the operating range of the devices. ${ }^{14}$

A more reliable approach would be to monolithically integrate all of the required optical functions into a single device. This could provide durable, long-lifetime compact systems for fluorescence detection. A very interesting example of this type of integrated fluorescence excitation and sensing systems was described recently by Thrush et al. in Ref. 16. The authors demonstrated how to obtain, in the same semiconductor substrate, a surface emitting laser, a photodetector, and an emission filter by exploiting the properties of vertical-cavity semiconductor devices. The laser was a vertical-cavity surfaceemitting laser (VCSEL), formed by a $p$-doped distributed Bragg reflector (DBR) reflector, an active region and a second $n$-doped DBR reflector, emitting at $773 \mathrm{~nm}$. This device was grown on the top of an intrinsic GaAs region, which was deposited on the top of $p$-doped GaAs substrate. A PIN photodetector was automatically created by removing from the wafer the top DBR and the active region. The $n$-doped DBR was used by the photodiode as electrical contact and emission filer. ${ }^{16}$ The major limitation of this type of devices is that they are available only in a limited spectral range, between 0.6 and $1 \mu \mathrm{m}$, while several fluorescent dyes have their operating wavelength in the blue-green region of the optical spectrum.

In this paper we describe an alternative approach to the monolithic integration of a light emitter and detector. The devices we have designed use a single $p-i-n$ junction so that the processing is kept simple. In this way using standard LED processing we have produced devices that act as excitation sources in forward bias and fluorescence detectors in reverse

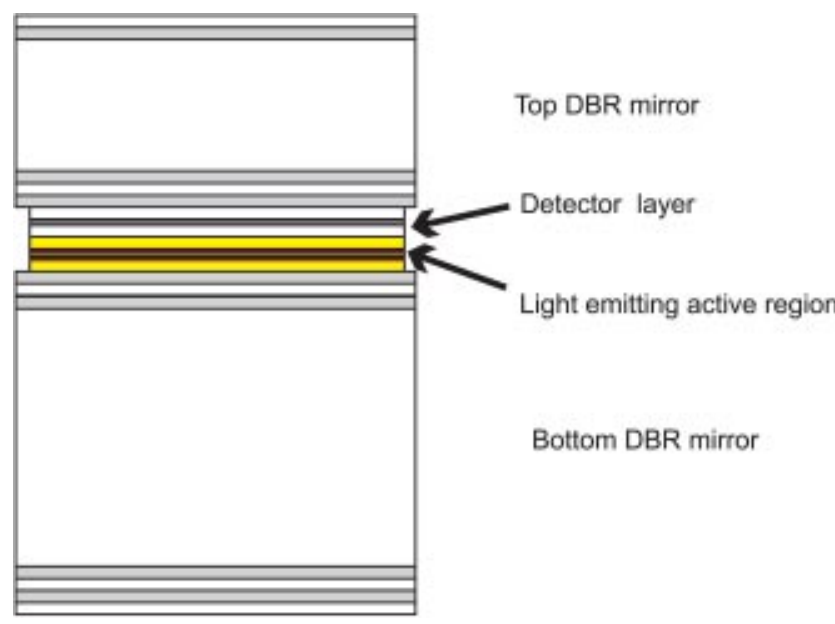

Fig. 1 Epilayer structure of the vertical-cavity semiconductor device.

bias. In the first part of this paper we describe the structure and the properties of these devices while in the second part we describe their performance in exciting and detecting fluorescence emission from a dye solution of volume in the order of a few microliters. The devices operate in the $650 \mathrm{~nm}$ wavelength range and so are suitable for dyes operating in the red region of the spectrum.

\section{Device Structure}

These devices are based on the generic structure of a resonant-cavity light-emitting diode (RCLED). The RCLED was demonstrated for the first time in 1991 by Schubert et al. ${ }^{17}$ and is basically a light emitting diode whose active region is placed within a resonant optical cavity to control and enhance its emission. ${ }^{18}$ The cavity is defined by a highly reflective mirror $(R=0.99)$ and a moderately reflective mirror $(R=0.9)^{17}$ that is used to outcouple the optical radiation. These mirrors are called DBRs and are made of several periods of alternating quarter wavelength $(\lambda / 4)$ layers of high and low refractive index semiconductor material. They produce wavelength dependent reflectivity by constructive interference $^{19}$ that can be tuned with the resonance of the cavity. The advantages that RCLEDs offer over conventional LEDs are improved directionality of the optical emission, ${ }^{17,18}$ better spectral purity, ${ }^{20}$ and enhanced external efficiency. ${ }^{21}$ These properties can be very useful to generate fluorescence with high efficiency in proximity of a microchannel.

The key concept embodied within our devices is the ability to both excite dye molecules and detect their redshifted fluorescence emission. To obtain this we fabricated a RCLED structure with a built-in photodetector layer in the device intrinsic region as can be seen in Fig. 1. The bottom DBR is composed of 32 pairs of $\lambda / 4$ layers of $\mathrm{Al}_{0.5} \mathrm{Ga}_{0.5} \mathrm{As} / \mathrm{AlAs}$, three GaInP/AlGaInP quantum wells form the light-emitting region, and eight pairs of $\lambda / 4$ layers of $\mathrm{Al}_{0.5} \mathrm{Ga}_{0.5} \mathrm{As} / \mathrm{AlAs}$ form the thin $p$-doped DBR. Above the light emitting region, in the intrinsic section of the device, a GaAs quantum well sandwiched between two $\mathrm{Al}_{0.5} \mathrm{Ga}_{0.5} \mathrm{As}$ layers is grown with total thickness equal to $3 / 4 \lambda$. The position of this detection layer is carefully chosen to be at a node in the standing wave formed by the electromagnetic field distribution inside the 


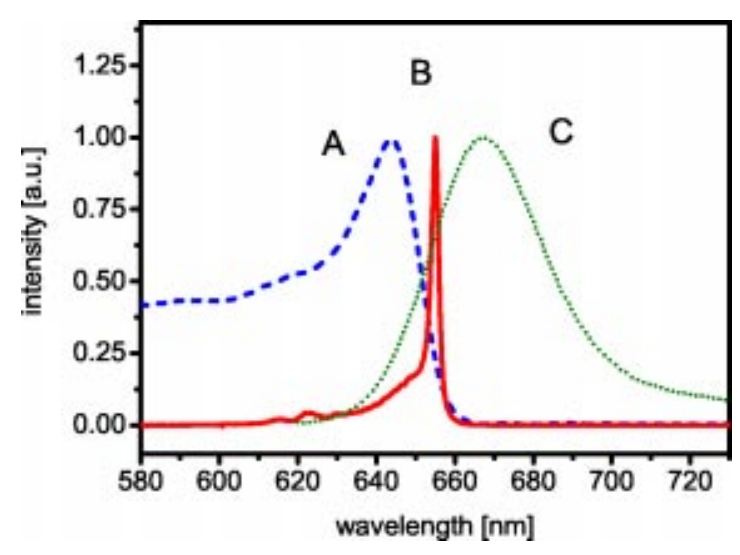

Fig. 2 Trace (a): normalized photovoltage absorption spectrum of a RCLED, trace (b): normalized emission spectrum of a RCLED, trace (c), normalized fluorescence emission spectrum of Cy5.

resonant cavity. ${ }^{22}$ In this way it is possible to introduce a detection layer inside the device without altering its spectral output and with minimum interaction between this additional layer and the emitted optical radiation. Thus long-wavelength fluorescence emission can be detected via photoabsorption without attenuating or altering substantially the excitation light produced within the device.

The main reason for using a separate GaAs quantum well (QW) detection layer can be understood with the help of Fig. 2. This figure shows a photovoltage absorption spectrum (a) and an emission spectrum (b) of a standard RCLED in normalized units to be compared with the fluorescence emission spectrum of Cy5 (c), a common dye in fluorescence spectroscopy $^{23}$ that we used in all our experiments. The absorption spectrum (a) shows that standard RCLEDs can always detect light when they are reverse biased but their peak wavelength responsivity lies on the blue side of the optical emission and the overlap with the fluorescence emission (c) is minimal. Furthermore the part of the optical spectrum between 640 and $660 \mathrm{~nm}$ has to be rejected in the detection stage because it contains contributions from scattering of the excitation source (b) and this cutoff makes a standard RCLED totally blind to fluorescence emission. The use of GaAs with its energy band gap of $1.424 \mathrm{eV}$ at room temperature allows for the broadening of the absorption spectrum into the infrared region so that the device becomes sensitive to wavelengths out to $870 \mathrm{~nm}$ and so covers the whole of the fluorescence emission.

A major problem related to the use of a single QW as photodetection layer is its narrow thickness $(15 \mathrm{~nm})$ that does not allow for a long interaction distance between the incident radiation and the absorber. In resonant-cavity enhanced photodetectors $^{24}$ a single or few QWs are used as active material and they are placed in correspondence of the maximum amplitude of the electric field in the cavity in a multipass detection scheme. Alternatively several QWs, up to 100, can be used as absorbers in high efficiency multiband gap solar cells, so that the large number of layers compensates for the short interaction distance. ${ }^{25}$ In our devices we were limited by the nonresonant position of the photoabsorber due to the requirement that it lies at a node of the electromagnetic field

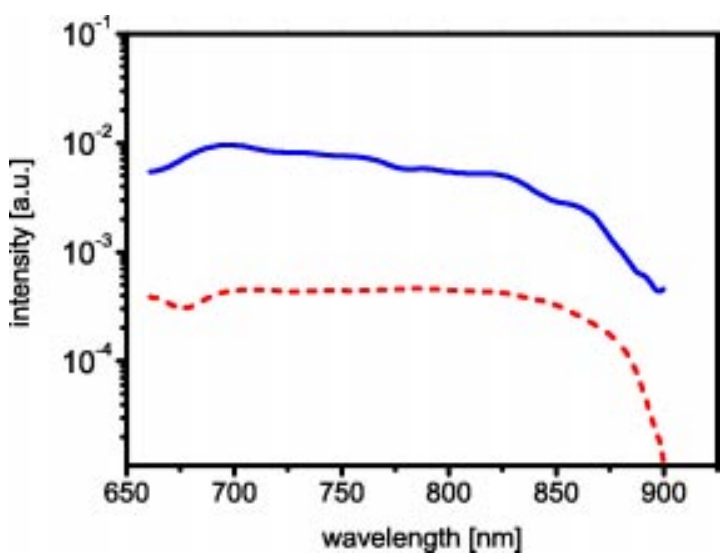

Fig. 3 Continuous line: photocurrent absorption spectrum of devices with integrated detector under $-5 \mathrm{~V}$ of reverse bias, dashed line: photocurrent absorption spectrum of the same device without reverse bias.

distribution inside the cavity to minimize the interaction with the emitted light.

By using only one GaAs QW as active absorber we were expecting to obtain very weak photocurrents. With our devices operated passively in detection mode we could measure photocurrents of the order of 1-5 nA when $1 \mathrm{~mW}$ of optical radiation from a $805 \mathrm{~nm}$ VCSEL was incident on their surface. However, by reverse-biasing these devices at sufficient high voltage, between 5 and $20 \mathrm{~V}$ depending on the transverse optical aperture, it was possible to operate them in avalanche mode $^{7}$ and the internal process of amplification increased the photogenerated current up to a few microamperes and the signal-to-noise ratio of $20 \mathrm{~dB}$. The reverse bias generates in the intrinsic region a high electric field per unit length, of the order of $1 \times 10^{5} \mathrm{~V} \mathrm{~cm}^{-1}$ that produces the internal amplification of the photogenerated electrons in the GaAs QW. Electrons in such a high electric field acquire a large kinetic energy sufficient to impact ionize other electrons bound to the lattice and create electron-hole pairs in the semiconductor. These particles also will be accelerated by the high electric fields present in the device and they further cause more impact-ionization events to produce an avalanche of impact ionization processes. In this way a single photon absorbed in the QW leads to a large number of electron-hole pairs and few photogenerated electrons can produce a large measurable current.

Figure 3 shows two photocurrent absorption spectra ${ }^{26}$ from a $50 \mu \mathrm{m}$ diameter device, one when the device is without reverse bias (dashed line) and the other (continous line) when $\mathrm{a}-5 \mathrm{~V}$ direct current $(\mathrm{dc})$ signal is applied between its anode and its cathode. It is possible to see clearly the effect of the internal amplification in the variation of the photocurrent signal. By applying a $-5 \mathrm{~V}$ dc reverse bias the signal is enhanced by $15 \mathrm{db}$ in all the spectral range where these detectors are sensitive and particularly where the dye used in our experiment has its peak wavelength emission. As described in Ref. 22, $10 \mu \mathrm{m}$ devices in detection mode with $13 \mathrm{~V}$ of reverse bias can detect incident optical radiation with intensity as low as $100 \mathrm{nW}$ at $805 \mathrm{~nm}$, an clear indication of their potential in detecting fluorescence emission at $670 \mathrm{~nm}$. The photocurrent noise level present in our devices operated in 


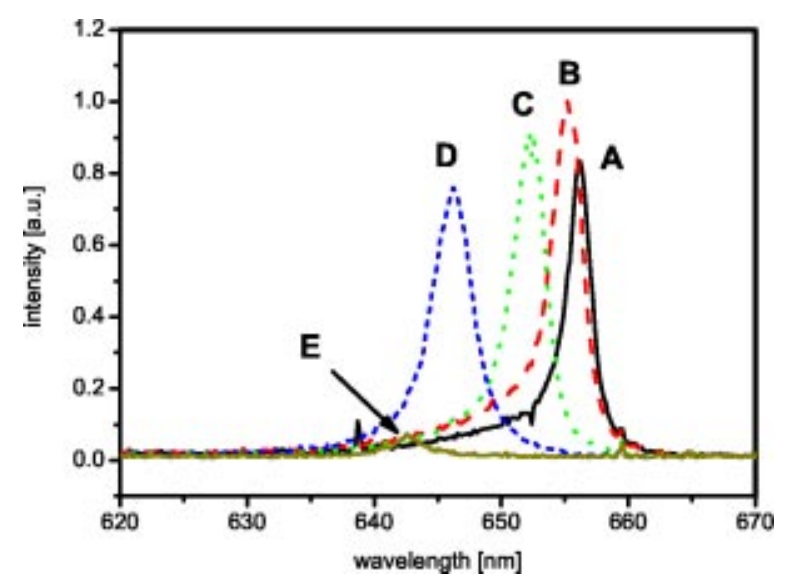

Fig. 4 RCLED emission spectra at different collection angles. Trace (a) corresponds to $0^{\circ}$, trace (b) to $10^{\circ}$, trace (c) to $20^{\circ}$, trace (d) to $40^{\circ}$ trace (e) $50^{\circ}$.

avalanche mode is one of the primary limitations for the detection of weak fluorescence signals. To overcome this problem it is necessary to use a lock-in amplifier or techniques to average the signal over a long time to obtain error free data.

An advantage that RCLEDs offer with respect to normal LEDs is that the presence of a resonant cavity with controllable thickness allows for a precise selection of their peak spectral emission. ${ }^{18}$ Thus RCLED can be designed to have their peak wavelength emission exactly resonant with the peak wavelength absorption of the fluorescence dye to increase the excitation efficiency. The spectral emission of RCLEDs can also be controlled after the growth of the complete vertical-cavity semiconductor structure. It has been demonstrated that the spectral properties of planar microcavities can vary substantially with the numerical aperture, ${ }^{27}$ thus the shape and the size of the optical aperture allows for a further control of the device spectral output.

Another RCLED property that makes these devices very suitable to generate fluorescence emission in microsystems is related to their angular emission spectra. ${ }^{28}$ This spectrum is determined by the action of the microcavity that defines a different resonant wavelength at each angle of observation. The spontaneous emission from the active region filtered by the cavity is enhanced at a different wavelength at different angles of observation and causes a shift of the peak output spectrum. The emission spectra of our devices were taken with a low numerical aperture (NA) objective lens $(\mathrm{NA}=0.12$ corresponding to an acceptance angle of $7^{\circ}$ ) placed in front of the device to collect light only in a limited emission cone. From Fig. 4 it can be seen that the peak spectral output shifts to shorter wavelengths as the angle of observation is increased while the spectral width at half maximum increases slightly. The total intensity of each spectrum, given by the area enclosed under the graph, remains of the same order of magnitude so that the optical power available to generate fluorescence does not decrease substantially as the light emission angle is increased.

From Fig. 4 it is possible to infer that the spectral components of the RCLED emission in the far field are also spatially separated. The spectra shown in this figure do not change if the device is rotated around its emission axis. The transverse cross section of the RCLED emission in the far field consists of circular rings where the optical output has the same intensity, the same wavelength and a similar spectral broadening. With spatial filtering techniques it is possible to select the spectral component of the RCLED resonant with the peak absorption wavelength of the dyes and most suitable for highly efficient fluorescence generation.

\section{Generation and Detection of Fluorescence Emission}

In a previous paper, ${ }^{22}$ we have demonstrated that these devices can detect optical radiation at wavelengths longer than that of their emission. By illuminating them with light from a $805 \mathrm{~nm}$ VCSEL and by operating them in avalanche mode we were able to detect a minimum flux of $10^{12}$ photons per second, a limitation mainly due to the high level of noise in the devices. In this paper we demonstrate that these devices can be used not only to detect optical radiation from a laser, but also to detect optical emission from a fluorescent sample. The fluorescent dye used during our experiments is a particular cyanine called Cy5, whose peak wavelength absorption is 648 $\mathrm{nm}$ and its peak wavelength emission $675 \mathrm{~nm}$. A brief introduction to fluorescence emission properties presented here will help to understand the experimental arrangement and the performance of our devices.

The phenomenon of fluorescence occurs in particular substances capable of absorbing optical radiation in a certain spectral range and emitting it redshifted at longer wavelength. The rate of fluorescence emission is defined to be equal to the rate of absorption multiplied by the quantum yield $Q$ of the fluorophore. Its expression is given by ${ }^{29}$

$$
I_{f}=Q\left[I_{i}\left(1-10^{\epsilon c d}\right)\right],
$$

where $I_{f}$ is the total fluorescence intensity in photons per unit time, $I_{i}$ is the intensity of the exciting light, in photons per unit time, $c$ is the concentration of the fluorophore, $d$ is the optical path length of the sample, $\epsilon$ is the molar extinction coefficient, and $Q$ is the quantum yield of the fluorophore. In very diluted solutions where only a small fraction of the incident light is absorbed the expression between round brackets in Eq. (1) can be simplified to obtain for the fluorescence emission rate

$$
I_{f}=Q\left[I_{i}(2.3 \epsilon c d)\right] .
$$

Equation (2) shows that only in diluted solutions the fluorescence intensity is proportional to the concentration $c$ of the dye. In this particular regime the fluorescence emission allows for a direct quantification of the number of dye molecules in the solution. The dye used in our experiment had a molar extinction coefficient $\epsilon$ equal to $2 \times 10^{5} \mathrm{M}^{-1} \mathrm{~cm}^{-1}$ and the path length $d$ of the cuvettes used in the experiments was 0.1 $\mathrm{cm}$. With these parameters a $50 \mu \mathrm{M}$ solution is the limit at which the linear approximation to obtain Eq. (2) is no longer valid and Eq. (1) has to be used to estimate the fluorescence emission. We have used samples with typical concentration of the order of $100 \mu \mathrm{M}$ in thin cuvettes to maximize the fluorescence output since our primary aim was simply to detect the presence of dye through its emission. In this regime it was not possible to quantify the concentration and particular attention 


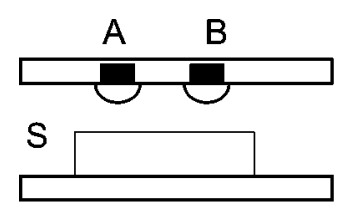

a)

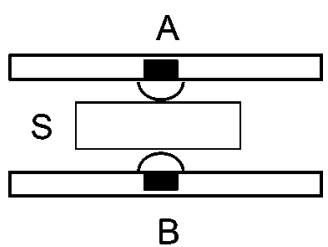

b)
Fig. 5 Two possible architectures for a microfluidic excitationdetection system. (a) is the excitation unit and (b) is the detection unit.

had to be payed to check that self-quenching effects would not have reduced the fluorescence output. The fluorescence emission increases with the fluorophore concentration up to a critical value $c^{*}$, after which it abruptly decreases with a further increase of the concentration. ${ }^{30}$ A detailed study on the photophysical properties of the cyanine dye Cy5 indicates that in the micromolar range of concentration self-aggregation and energy transfer can affect the dye output ${ }^{31}$ in an undesirable way for our purposes. Cy5 has been used by Roulet et al. in Ref. 32 to test a microlens system they developed for fluorescence detection and they used this dye in concentrations up to $50 \mu \mathrm{M}$ successfully in their chemical microsystems. Following these results we were quite confident that operating with $100 \mu \mathrm{M}$ solutions we were still in a regime where selfquenching effects were not dominant. Further developments of these devices could increase their sensitivity and reduce their internal noise to enable them to detect fluorescence emission from more dilute solutions.

The main practical problem to face in a basic experiment for the detection of fluorescence emission concerns the choice of the directions for sample illumination and light collection. The approaches to illuminate and observe fluorescence samples in a cuvette are (a) right-angle method, where the fluorescence emission is collected in a direction orthogonal to that of excitation, (b) frontal method where the fluorescence emission is collected from the front of the cuvette by tilting it or putting a beam splitter between the cuvette and the light source, and (c) straight through method where the fluorescence emission is collected in the same direction of the excitation source. ${ }^{29}$ The most used method in analytical instrumentation is the right-angle one, useful to avoid contributions from the excitation light to the measured total intensity and suitable for diluted and weakly absorbing solutions. The main purpose of the frontal and straight through arrangements is to characterize emission spectra of highly concentrated solutions of fluorescent dyes and they need dichroic beam splitters and/or strong passband optical filters to cutoff the emission from the pump source.

There are two possible microfluidic platform architectures where these devices could be successfully employed, as shown in Fig. 5. In configutation (a), as described in Ref. 16, a device operating as emitter and a device operating as detector lay on the same side of the microchannel and the backscattered fluorescence emission has to be detected by the sensor. A system of lenses or optical waveguides can guide the excitation source output onto the target and direct its fluorescence emission onto the detector with minimal overlapping of the two optical emission. In configuration (b) instead two de- vices are mounted on opposite sides of the microchannel and a system of microlenses can focus the excitation light onto the sample to be tested and collect its fluorescence emission. In this case excitation light and fluorescence emission can overlap spatially to become indistinguishable. Roulet et al. in Ref. 32 have demonstrated a system of microlenses that can decouple excitation light from fluorescence emission in the straight-on direction. This type of architecture is a suitable platform where our devices could be successflully used. The advantage that our devices offer in this case is the possibility to produce a single unit that can be used either as emitter or as detector, thus minimizing and simplifying the semiconductor processing steps. Emission and detection units can be fabricated in large matrices with standard semiconductor fabrication techniques for highly parallel detection systems such as the biochips described in Ref. 1.

We chose the straight-on geometry to test the performance of our devices because of the possibility of maximizing the light collection efficiency with only one lens. A long wavelength passband filter had to be inserted to mimic the separation of excitation light and fluorescence emission. The output of a RCLED is highly directional with a cylindrical symmetry and with these devices it is possible to generate disk-shaped fluorescence emission easy to couple into the detectors or into an optical fiber. Given a quantum yield $Q$ of 0.3 for Cy5 and using Eq. (1) it is possible to estimate that, with about $5 \mu \mathrm{W}$ of resonant optical radiation to excite the dye, 200-300 nW of fluorescence emission can be produced in the straight through direction. This amount of optical power could be easily detected by our devices.

There are facts to be taken into account carefully when the fluorescence emission is collected with the straight-through configuration in highly concentrated samples. As stated before, in these operating conditions the fluorescence emission is not proportional to the concentration of the sample and to its molar extinction coefficient, so these two quantities cannot be evaluated in this type of experiment. Furthermore the excitation and emission spectra after the cuvette can be distorted and self-absorption of fluorescence can reduce drastically the fluorescence output. Hence, the fluorescence output from long optical path cuvette containing a highly concentrated solution can be zero. In this type of configuration it is very important to consider the presence of the so called inner-filter effects. ${ }^{29}$ These effects can be due to the absorption of some of the excitation light before it reaches the point inside the cuvette where luminescence occurs (primary inner filter effect) or can be due to reabsorption of some of the fluorescence emission before it leaves the cuvette. ${ }^{33}$ These inner-filter effects change drastically the output from the cuvette but can have also the beneficial effect of eliminating part of the excitation light in the straight-through direction of observation.

The experimental setup used to generate and detect fluorescence emission with our devices is shown in Fig. 6 . A $5 \times$ or $10 \times$ microscope objective lens $L_{1}$ was put in front of the device to collected light in a limited emission cone and to focus it on a small transverse section of the cuvette $C$. The device $D_{1}$ could be rotated to select the angle at which its peak wavelength emission was resonant with that of the dye absorption to maximize the fluorescence generation efficiency. A long wavelength passband filter $F$ inserted between the cuvette $C$ and the second lens $L_{2}$ cutoff the optical emis- 


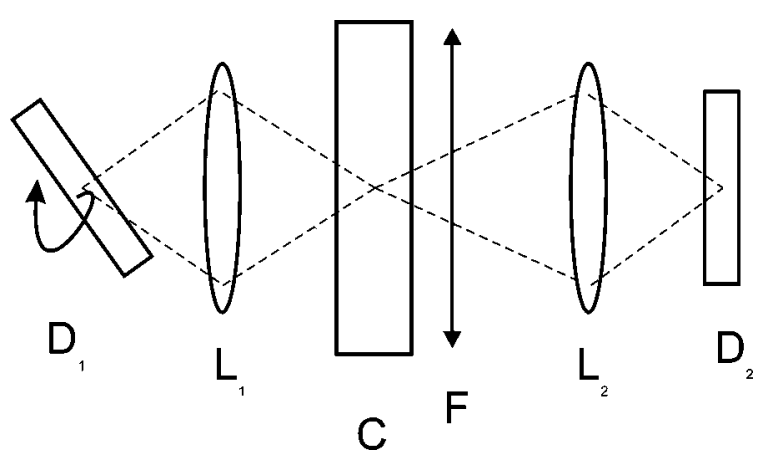

Fig. 6 Experimental setup used for the characterization of the fluorescence emission and the response of the device with integrated detector. $D_{1}$ and $D_{2}$ are, respectively, the excitation device and the detecting device, $L_{1}$ and $L_{2}$ are microscope objectives, $C$ is the cuvette, and $F$ is the long-pass optical filter.

sion at all wavelengths shorter than $665 \mathrm{~nm}$. The fluorescence emission that passed through the filter was collected by the second lens $L_{2}$, a $40 \times$ objective lens with numerical aperture equal to 0.65 , and focused onto a second device or into an optical fiber to be measured. The pump source was slowly modulated at about $1 \mathrm{kHz}$ so that the presence of the fluorescence signal could be revealed in the detector photocurrent with an oscilloscope or measured precisely with a lock-in amplifier.

The setup was kept as small and compact as possible to avoid losses by diffraction. A typical distance between the excitation device and the detection device was $10 \mathrm{~cm}$. The cuvette path length had to be kept as short as possible, 1-2 $\mathrm{mm}$ or less, to avoid inner-filter effects canceling out the fluorescence emission. By using this particular setup we always checked that the emission from the excitation source was completely cutoff by the passband filter and that the fluorescence emission was not distorted by inner-filter effects or by Raman scattering. The small transverse size of a RCLED allowed us to excite a small volume of a Cy5 aqueous solution. The beam waist diameter of the incident radiation was usually less than $2 \mathrm{~mm}$ and produced an illuminated volume of the order of $5 \mu \mathrm{L}$. The rotation of the emitting device $D_{1}$ allowed us to select the part of the RCLED angular emission spectrum resonant with the absorption of $\mathrm{Cy} 5$ to generate fluorescence emission in the most efficient conditions. Figure 7 shows some of the spectra of the signals used to excite the dye and to produce fluorescence emission shown in Fig. 8. These signals were peaked respectively at 638,639, and $644 \mathrm{~nm}$ and they were obtained by rotating the device $D_{1}$ in front of the lens of $44^{\circ}, 43^{\circ}$, and $35^{\circ}$. The RCLED output was focused on a multimode optical fiber coupled to an optical spectrum analyser, ANDO AQ6315, to be analyzed. The device output power incident on the optical fiber in the three different configurations was $0.8 \mu \mathrm{W}$ at $638 \mathrm{~nm}, 2.1 \mu \mathrm{W}$ at $639 \mathrm{~nm}$, and $3.25 \mu \mathrm{W}$ at $644 \mathrm{~nm}$.

The optical power available from our devices was quite low, typically of the order of a few microwatts, because the selection of a portion of the emitted pattern. Despite operating in a tilted configuration, the spectral output remained quite a narrow band, less than $20 \mathrm{~nm}$, and the tails at long wavelength of the selected spectra did not overlap significantly with the

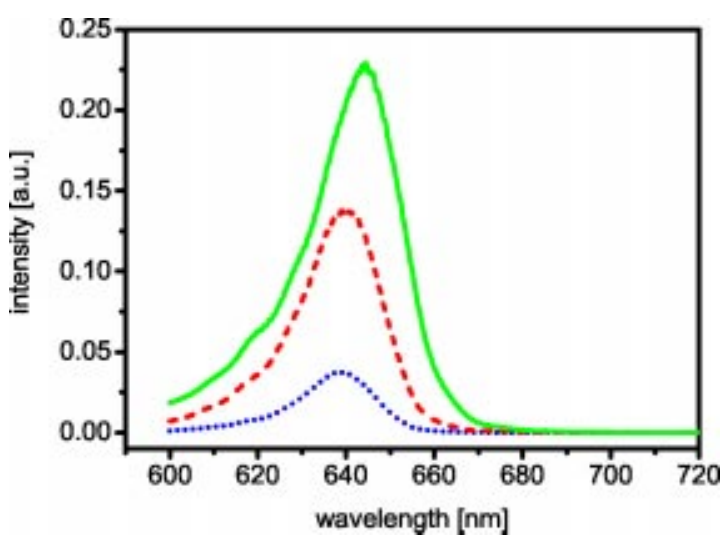

Fig. 7 RCLED emission spectra used for dye excitation. Continuous line, emission at $35^{\circ}$, dashed line, emission at $41^{\circ}$, and dotted line emission at $44^{\circ}$.

fluorescence emission. Optical emission from RCLED collected at angles smaller than $35^{\circ}$ would have given a weak contribution at $680 \mathrm{~nm}$ that could be comparable to the fluorescence emission and for this reason the collection at these angles was discarded. With a $100 \mu \mathrm{M}$ solution of Cy5 in pure water we could produce undistorted fluorescence emission in the straight-through direction of observation with a total power of 100-200 $\mathrm{nW}$ easy detectable by our devices in avalanche mode. The confirmation that we were producing fluorescence from the Cy5 diluted in water was the presence of a peak at $674 \mathrm{~nm}$ in the emission spectra that did not change as the intensity of the excitation source was increased, as can be seen from Fig. 8. The presence of strong inner-filter effects is clearly visible in the central trough of these spectra around $647 \mathrm{~nm}$ where the excitation light is totally absorbed in the cuvette. The part of the optical spectrum on the left side of Fig. 8 peaked at about $620 \mathrm{~nm}$ is due to part of the excitation source that could to pass through the cuvette without being altered by the fluorophore.

By using a Schott RG665 long-wavelength passband filter between the cuvette and the lens $L_{2}$ the cutoff of the $620 \mathrm{~nm}$ emission was performed very efficiently, as can be seen in Fig. 9. The two spectra shown in this figure demonstrate the

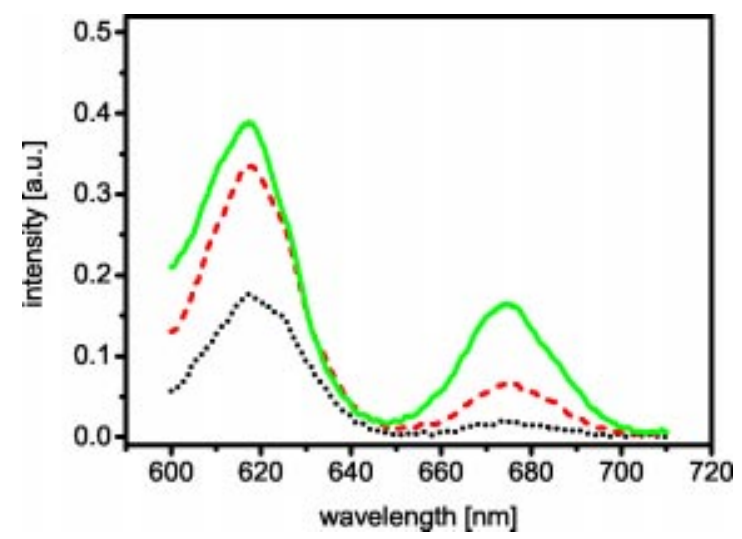

Fig. 8 Optical emission spectra of the solution of Cy5 with different incident optical power $P_{0}$. Contininuous line, $P_{0}=3.25 \mu \mathrm{W}$, dashed line, $P_{0}=2.1 \mu \mathrm{W}$, and dotted line $P_{0}=0.8 \mu \mathrm{W}$. 


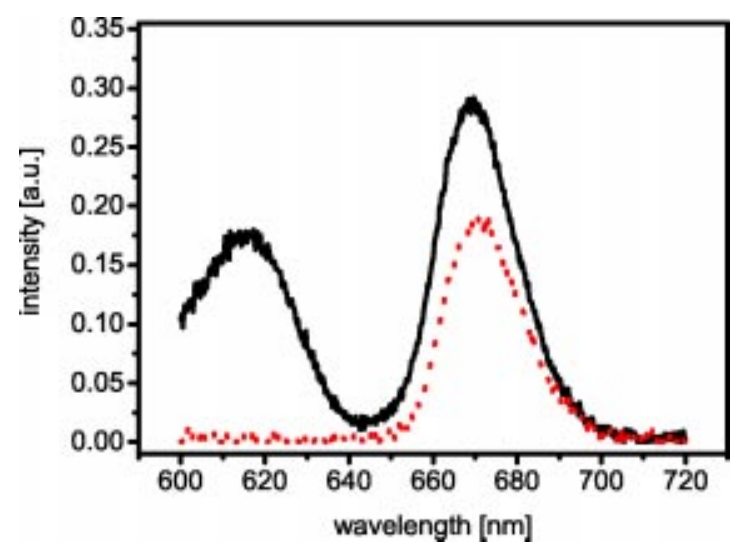

Fig. 9 Emission spectra from the Cy5 solution in the cuvette without an optical filter, continuous line, and with optical filter dotted line.

efficiency of this optical filter in eliminating unwanted optical radiation radiation at wavelengths shorter than $665 \mathrm{~nm}$. It is extremely important to remove all optical radiation at wavelengths shorter than $648 \mathrm{~nm}$ because the presence of the resonant cavity strongly coupled to the active medium for the light emission makes these devices very sensitive when acting as detectors in this wavelength range.

We demonstrated that these devices can detect fluorescence emission by recording with an oscilloscope (Tektronix TDS 2014) the photocurrent signal generated by a device in detection mode with the same frequency as that of a modulated device used as excitation source. Figure 10 shows some time traces of the detector response taken with the oscilloscope in average mode with number of acquisitions equal to 256. Trace (a) represents the signal generated by the device in photodetection mode in absence of the optical passband filter and trace (b) represents the same signal when the optical passband filter is inserted. The difference in magnitude between the two traces shows how critical is the elimination of optical radiation at wavelengths shorter than $648 \mathrm{~nm}$ that is efficiently detected by our devices but is not related to fluorescence emission.

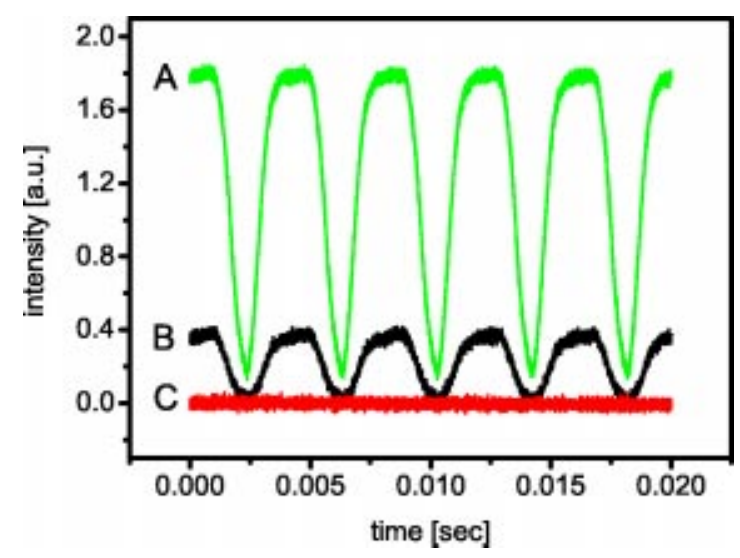

Fig. 10 Modulation signal from a device with integrated detector without an optical filter, trace (a) and with an optical filter, trace (b). The base line in the signal from the detector when the light from the cuvette was blocked.

\section{Conclusions}

We have described in detail the properties of a new type of integrated semiconductor device capable of generating efficiently fluorescence emission in small volumes and detecting it when its total power is of the order of a few hundred nanowatts. We have described their particular structure originated by the idea of integrating the three basic functions of a microfluorimeter and keeping the processing needed to make them as simple as possible. The features of the RCLED allows for the production of devices whose optical emission is resonant with the maximum absorption of the fluorophore to maximize the efficiency of the generation of fluorescence emission. The insertion of a single GaAs QW in the intrinsic region of these devices makes them capable of detecting fluorescence at wavelengths longer than that of their emission. By using avalanche multiplication of the photogenerated electrons a signal of the order of microamperes can be obtained from a single QW detector whose output would otherwise be extremely weak. After external optical filtering of the emission from a cuvette containing a concentrated solution of $\mathrm{Cy} 5$, a weak fluorescence emission has been detected with an experimental arrangement made to mimic as close as possible the behavior of a small plug of fluorophore in a microchannel or of a single assay in an optical biochip. These devices are the result of a preliminary project whose final goal is to integrate in the same semiconductor structure an optical filtering system to reject unwanted excitation light that can overlap with the fluorescence emission. The realization of such a device will allow for the monolithic integration in the same devices of the three functions of a microfluorimeter.

\section{Acknowledgments}

The authors would like to thank the Engeneering and Physical Science Research Council (EPSRC) for the financial support of this project and IQE Plc for the wafer growth.

\section{References}

1. T. Vo-Dinh, "Biochips and microarrays: tools for the new medicine," in Biomedical Photonics Handbook, T. Vo-Dinh, Ed., Chap. 51, pp. 51.1-51.29, CRC Press, Boca Raton (2003).

2. A. Manz, N. Graber, and H. M. Widmer, "Miniaturized total chemical analysis systems: a novel concept for chemical sensing," Sens. Actuators B 1, 244-248 (1990).

3. J. Roulet, R. Volkel, H. P. Herzig, E. Verpoorte, N. F. de Rooij, and R. Dandliker, "Performance of an integrated microoptical system for fluorescence detection in microfluidic systems," Anal. Chem. 74, 3400-3407 (2002).

4. J. R. Webster, M. A. Burns, D. T. Burke, and C. H. Mastrangelo, "Monolithic capillary electrophoresis device with integrated fluorescence detector," Anal. Chem. 73, 1622-1626 (2001).

5. J. R. Lakowicz, "Instrumentation for fluorescence spectroscopy," in Principle of Fluorescence Spectroscopy, Chap. 2, pp. 25-61, Plenum, New York (1999).

6. B. W. Smith, B. T. Jones, and J. D. Winefordner, "High-precision fluorimetry with a light-emitting diode source," Appl. Spectrosc. 42, 1469-1472 (1988).

7. S. O. Kasap, "Photodetectors," in Optoelectronics and Photonics: Principles and Practices, Chap. 5, pp. 230-234, Prentice Hall, Upper Saddle River, NJ (2001).

8. O. S. Wolfbeis, B. P. H. Schaffar, and E. Kaschnitz, "Fibre-optical titrations 3, construction and performance of an acid-base titrator with a blue led as a light source," Analyst (Cambridge, U.K.) 111, 1331-1335 (1986).

9. P. K. Dasgupta, I. Eom, K. J. Morris, and J. Li, "Light emitting diode based detectors absorbance, fluorescence and spectroelectrochemical 
measurements in a planar flow-through cell," Anal. Chim. Acta 500, 337-364 (2003).

10. T. Araki and H. Misawa, "Light-emitting diode based nanosecond ultraviolet light source for fluorescence lifetime measurements," Rev. Sci. Instrum. 66, 5469-5472 (1995).

11. J. Sipior, G. M. Carter, J. R. Lakowicz, and G. Rao, "Single quantum well light emitting diode demonstrated as excitation sources for nanosecond phase-modulation fluorescence lifetime measurements," Rev. Sci. Instrum. 67, 3795-3798 (1996).

12. J. Sipior, G. M. Carter, J. R. Lakowicz, and G. Rao, "Blue light emitting diode demonstrated as ultaviolet excitation source for nanosecond phase-modulation fluorescence lifetime measurements," Rev. Sci. Instrum. 68, 2666-2670 (1996).

13. M. L. Chabinyc, D. T. Chiu, J. C. McDonald, A. D. Stroock, J. F. Christian, A. M. Karfer, and G. M. Whitesides, "An integrated fluorescence detection system in poly(dimethylsiloxane) for microfluidic application," Anal. Chem. 73, 4491-4498 (2001).

14. D. Fehly, A. Schlachetzki, A. S. Bakin, A. Guttzeit, and H. Wehmann, "Monolithic ingaasp optoelectronic devices with silicon electronics," IEEE J. Quantum Electron. 37, 1246-1252 (2001).

15. J. A. Chediak, Z. Luo, J. Seo, N. Cheug, L. P. Lee, and T. D. Sands, "Heterogeneous integration of cds filters with gan leds for fluorescence detection microsystems," Sens. Actuators, A 111, 1-7 (2004).

16. E. Thrush, O. Levi, W. Ha, G. Carey, L. Cook, J. Deich, S. J. Smith, W. E. Moerner, and J. S. Harris, "Integrated semiconductor veritcalcavity surface-emitting lasers and pin photodetectors for biomedical fluorescence sensing," IEEE J. Quantum Electron. 40, 491-498 (2004).

17. E. F. Schubert, Y. H. Wang, A. Y. Cho, L. W. Tu, and G. J. Zydzik, "Resonant cavity light-emitting diode," Appl. Phys. Lett. 60, 921923 (1992).

18. G. Bjork, S. Machida, Y. Yamamoto, and K. Igeta, "Modification of spontaneous emission rate in planar dielectric microcavity structures," Phys. Rev. A 44, 669-681 (1991).

19. H. Benitsy, H. D. Neve, and C. Weisbuch, "Impact of planar microcavity effects on light extraction-part i: basic concepts and analytical trends," IEEE J. Quantum Electron. 34, 1612-1631 (1998).

20. N. E. Hunt, F. E. Schubert, R. A. Logan, and G. J. Zydzik, "Enhanced spectral power density and reduced linewidth at $1.3 \mu \mathrm{m}$ in an ingaasp quantum well resonant-cavity light-emitting diode," Appl. Phys. Lett. 61, 2287-2298 (1992).
21. H. D. Neve, J. Blondelle, P. V. Daele, P. Demeester, R. Baets, and G. Borghs, "Recycling of guided mode light emission in planar microcavity light emitting diodes," Appl. Phys. Lett. 70, 799-781 (1997).

22. P. A. Porta and H. D. Summers, "Vertical-cavity semiconductor devices for fluorescence generation and detection on a chip," Appl. Phys. Lett. 85, 1889-1891 (2004).

23. G. Jiang, S. Attiya, G. Ocvirk, W. E. Lee, and D. J. Harrison, "Red diode laser induced fluorescence detection with a confocal microscope on a microchip for capillary electrophoresis," Biosens. Bioelectron. 14, 861-869 (2000).

24. K. Kishino, M. S. Unlu, J. I. Chyi, J. Reed, L. Arsenault, and H. Morkoc, "Resonant cavity enhanced (rce) photodetectors," IEEE J. Quantum Electron. 27, 2025-2034 (1991).

25. K. W. J. Barnham and G. Duggan, "A new approach to highefficiency multi-band-gap solar cells," J. Appl. Phys. 67, 3490-3493 (1990).

26. P. Blood, "Measurement of optical absorption in epitaxial semiconductor layers by a photovoltage method," J. Appl. Phys. 58, 22882295 (1985).

27. R. F. Oulton, J. W. Gray, P. N. Stavrinou, and G. Parry, "Insight into planar microcavity emission as a function of the numerical aperture," Opt. Commun. 195, 327-338 (2001).

28. P. N. Stavrinou, M. Whitehead, G. Parry, and C. C. Button, "Angular spectrum of visible resonant cavity light-emitting diodes," J. Appl. Phys. 86, 3475-3477 (1999).

29. C. A. Parker and W. T. Rees, "Fluorescence spectrometry: a review," Analyst (Cambridge, U.K.) 87, 83-111 (1962).

30. S. Hamann, J. F. Kiilgaard, T. Litman, F. J. Alvarez-Leefmans, B. R. Winther, and T. Zeuthen, "Measurements of cell volume changes by fluorescence self-quenching," J. Fluoresc. 12, 139-145 (2002).

31. J. Widergren and P. Schwille, "Characterization of photoinduced isomerization and backisomerization of the cyanine dye cy5 by fluorescence correlation spectroscopy," J. Phys. Chem. 104, 6416-6428 (2000).

32. J. C. Roulet, R. Volkel, H. P. Herzig, E. Verpoorte, N. F. de Rooij, and R. Dandliker, "Microlens system for fluorescence detection in chemical microsystems," Opt. Eng. 40, 814-821 (2001).

33. M. Kubista, R. Sjoback, S. Eriksson, and B. Albinsson, "Experimental correction for the inner-filter effect in fluorescence spectra," Analyst (Cambridge, U.K.) 119, 417-419 (1994). 